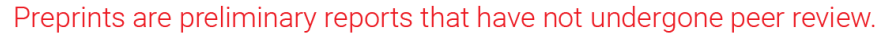 They should not be considered conclusive, used to inform clinical practice, or referenced by the media as validated information. \\ Clinical Characteristics and Prognostic Nomogram in Patients With Pineoblastoma
}

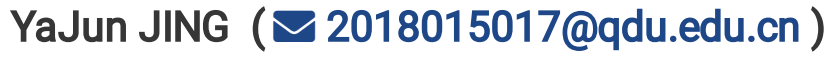

The affiliated hospital of Qingdao University https://orcid.org/0000-0002-1291-4104

\section{WenShuai DENG}

The affiliated hospital of Qingdao University

\section{YunXia JIANG}

Qingdao University

\section{MingXia BI}

Medical college of Qingdao University

\section{MingChao FAN}

The affiliated hospital of Qingdao University

\section{YiJun GAO}

Medical college of Qingdao University

\section{Peng Sun}

The Affiliated Hospital of Qingdao University

\section{Research article}

Keywords: Pineoblastoma, Cox regression analysis, Nomogram, Risk factor,Survival

Posted Date: December 8th, 2020

DOl: https://doi.org/10.21203/rs.3.rs-121120/v1

License: (c) (1) This work is licensed under a Creative Commons Attribution 4.0 International License.

Read Full License 


\section{Abstract}

Background: Pineoblastoma (PB) is an infrequent entity of the central nervous system. The data about clinical outcomes of PB is exceedingly limited due to its rarity. Notably, the optimal treatment approaches and prognostic factors on PB is still unclear. Thus the aims of this study are to identify prognosisassociated factors and develop a predictive nomogram of PB.

Method: Data of 243 patients with PB ( $\leq 29$ years), collected by the Surveillance, Epidemiology, and End Results database, were randomly carved up the primary $(n=172)$ and validation cohort $(n=71)$ two groups. A prognostic nomogram was developed based on primary cohorts and optimized via the Akaike Information Criterion. Calibration curves were applied to show the results of validation (internal, external, and cross-validation). Additionally, its predictive performance was evaluated by the concordance index and receiver operating characteristic curve. Finally, decision curve analyses were employed to assess its clinical utility.

Results: Age, year of diagnosis, treatment, tumor size, and tumor extension were identified as independent predictors of PB based on multivariate Cox regression analysis. The model presented an excellent discriminating ability (concordance index of the nomogram: $0.805 ; 95 \%$ confidence interval: $0.78-0.83$; area under the receiver operating characteristic curve with a range from 0.7 to 0.9 ). The calibration plots (probability of survival) is consistent with the results predicted by the nomogram. The results of the decision curve analysis showed that the nomogram has potential clinical applicability.

Conclusion: The nomogram can be used to assess prognosis and determine appropriate treatment options.

\section{Background}

The pineal gland is an endocrine gland located in the midline of the brain, which modulates indirectly circadian rhythms via secreting melatonin. Some primary tumors growing out of there are termed as the pineal parenchymal tumors (PPTs), which are considered as rare neoplasms and account for less than $1 \%$ of all primary central nervous system tumors (1). Interestingly, there, for these tumors, is a higher incidence in groups of infants and adolescents than adults $(2,3)$. Additionally, the varied growth patterns and histological features were typically exhibited in different histological types of PPTs. PPTs were classified into the pineocytoma (ICD-0 9361/1, WHO grade I), PPT of intermediate differentiation (ICD-O 9362/3, WHO grade II/III), pineoblastoma (PB) (ICD-O 9362/3, WHO grade IV), and papillary tumor (ICD-0 $9395 / 3$, WHO grade II/III) four significant subgroups in the classification of tumors of the central nervous system published by the in 2016 (4). Of those, PB covers a substantial proportion of PPTs, with up to 25$50 \%$ (5), and a high incidence of PB is observed in children, adolescents, and young adults (6-8).

PB is defined as a high-grade malignant primary supratentorial neuroectodermal tumor in the central nervous system (9). Similar to other malignancies, the patients with PB harbors traditionally exceedingly poor prognosis on account of the aggressiveness of PB. Besides, a high recurrence rate and strong 
inclination of metastasis, especially systemic metastasis, are also one of the main reasons why PB exhibits a worse prognosis. It was reported by the published literature that PB manifested a predilection for seeding along the craniospinal axis and metastasizing to parts other than the central nervous system $(10,11)$, such as lung (12), bone (13-16), and peritoneum (17). However, the data on the survival of patients with PB is extremely limited, owing to the rarity of PB cases. Up to date there were hundreds of literature to report PB, but most as single case report or in small series (18-20). Although those studies, at some level, were a valuable source of information concerning PB, some reliable medical proofs for treatment decision-making were not yet supplied by those with inherent drawbacks, such as small sample numbers and inadequate statistical power. Therefore, there is thus far no yet clearly defined independent prognostic factors of PB and generally accepted treatment regiments.

To address these shortcomings, we conducted a retrospective study in a large series with 243 PB cases from the Surveillance, Epidemiology, and End Results (SEER) database. Firstly, the Cox proportional hazard regression analysis was applied to identify the potential factors and independent prognostic factors of PB. Secondly, survival outcomes of patients with PB were compared based on the independent prognostic factors of PB. Finally, the nomogram model was established based on independent prognostic factors to provide a more accurate prediction of the survival of patients with PB.

\section{Methods}

\subsection{Data resource}

The data of 243 PB cases were acquired from the SEER database by the SEER *Sat version 8.3.6 (https://seer.cancer.gov/), which collected the patient demographics, diagnosis years, tumor characteristics (tumor size, grade, and extension), course of treatment, and follow-up of vital status.

\subsection{Inclusion and exclusion criteria}

To meet research requirements, we initially set up the inclusion and exclusion criteria for sample selection in this study. Inclusion criteria: $₫$ patients were diagnosed as PB with the ICD-0-3 code 9362/3; $₫$ year of

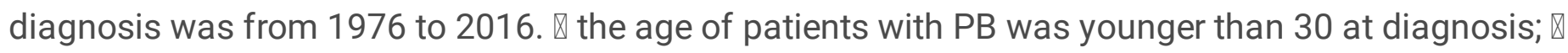

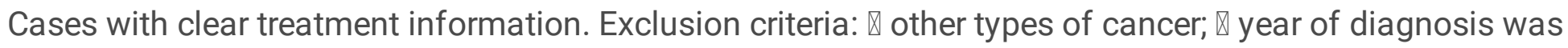
before 1976 and after 2016; 『patients with more than 30 years of age; $\otimes$ patients with unclear treatment information (unknown); $\Downarrow$ dead of causes other than PB; $\Downarrow$ single cases in some items (such as the RT prior to surgery, intraoperative beam radiotherapy [RT], RT before and after surgery as well as sequence unknown, but both were given). Ultimately, 243 patients with PB were selected for this analysis (Fig. 1).

\subsection{Variables selection}

The age, sex, race, year of diagnosis, laterality, therapy, tumor extension, and tumor size were included and analyzed in this study. Several of those factors often had various subclasses, such as laterality (including right/lift-origin of primary and paired site, but no information concerning laterality), therapy 
(comprising only biopsy; surgical treatment; RT; chemotherapy [CT]; RT after surgery; RT combined with $\mathrm{CT}$, sequence unknown; and RT after non-primary surgical procedure combined with $\mathrm{CT}$, sequence unknown), extent of tumor extension (including invasive tumor confined to gland of origin, localized, not otherwise specified, adjacent connective tissue, adjacent organs/structures, and further contiguous extension, http://web2.facs.org/cstage0205/intracranialgland/IntracranialGland_bfy.html), and tumor size. Survival time was defined as the period from diagnosis to death or last follow-up, and those cases lost to follow-up were censored by the SEER. Non-survival included death caused by PB. Additionally, in view of existing incomplete items (including tumor extension and size), the missing value was replaced by a computer-generated

data, which was achieved via methods of multiple imputations by the R software, which could boost case number and statistical power (21).

\subsection{Statistical analysis}

All cases were partitioned at random into the training and validation cohort two groups in proportion to 7:3 (22). The data from the training cohort was applied to establish the predictive model and nomogram, while the validation cohort was employed to the external validation of the model. The specific process for the construction of the predictive model and nomogram was as follows. Firstly, we explored the possible

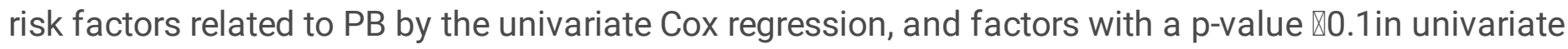
Cox regression were defined as the potential risk factors of PB. Secondly, all possible risk factors were selected and analyzed in the multivariate Cox regression, and those with a p-value $<0.05$ in the multivariate analysis were named as independent predictors of PB. Thirdly, the predictive model was developed based on all independent predictors, and the model was optimized and visualized by virtue of the Akaike Information Criterion (AIC) and nomogram, respectively (23).

The internal and external validation was carried out to validate the accuracy of the model in the training and validation cohort, respectively. Furthermore, we also performed cross-validation in both the cohort by bootstrapping. Besides, the accuracy and distinguishing ability of the model were assessed by the timedependent receiver operating characteristic (ROC) curve and concordance index (C-index) with bootstrapping $(24,25)$. The calibration curves were applied to compare the association and deference between the actual observations and predicted outcomes of the model (26). Finally, we evaluated the clinical utility of the model by the decision curve analysis (DCA) (27).

In this study, all continuous variables (including age, years of diagnosis, and tumor size) were transformed into the categorical variables according to the best cut-off values, which were identified by the X-title software (28). The definition of the optimal cut-off value was the point, which was the most significant split among the survival distributions of those factors with different categorical ages and tumor sizes (22). Last of all, the new categorical variables were regarded as the predictors which were analyzed in the survival analysis. 
The R (http://www.r-project.org/; version 3.6.3) and X-title software (Yale University, New Haven, CT, USA;

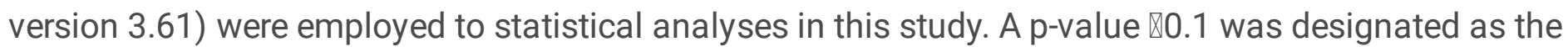
criterion for excluding a variable from the multivariate Cox proportional hazards model, and a $p$-value < 0.05 denoted statistical significance for all other tests.

\section{Results}

\subsection{Clinical and pathological characteristics of patients}

In this study, 243 patients with PB were split into the training cohort $(\mathrm{n}=172)$ and the validation cohort $(n=71)$. Table 1 shows baseline information about patients. The majority of patients were white $(n=163$, $67.08 \%)$ and age $\otimes 5$ years $(n=66,27.16 \%)$. Besides, most patients did not have paired sites due to the presence of lateral features $(n=239,98.35 \%)$. There is no other indication that the tumor can only extend to the glands and localities of origin $(n=142,54.32 \%)$. From 2003 to 2016 , about $80 \%$ of patients were diagnosed with disease $(n=157,64.61 \%)$. Surprisingly, in terms of histology, the rate of confirmed cases was as high as $97 \%(n=239,98.35 \%)$.

\subsection{Univariate analysis}

The age, year of diagnosis, treatment (only biopsy, Surgery, RT, CT, RT after surgery, RT and CT, RT after surgery combined with $\mathrm{CT}$ ), tumor size, and tumor extension were identified as the possible risk factors of PB in this study, which were statistically significant with the survival (Pख0.05) (Figure 2). The rest of the factors with $\mathrm{P} \otimes 0.05$ in univariate analysis excluded from multivariate analysis.

\subsection{Independent prognostic factors in the training cohort}

As is shown in figure 3, the age, sex, therapy, tumor extension, and tumor size were identified as the independent prognostic factors of PB. In details, the age (hazard ratio $[\mathrm{HR}]=0.72, \mathrm{P} \llbracket 0.05$ ), therapy options (RT combined with CT: HR=0.17, P凶0.001; RT after surgery: $\mathrm{HR}=0.38, \mathrm{P}<0.05 ; \mathrm{RT}$ after surgery combined with $\mathrm{CT}: \mathrm{HR}=0.30, \mathrm{P}<0.05$ ), extent of tumor extension (localized, NOS: $\mathrm{HR}=3.05, \mathrm{P} \otimes 0.005$; extension adjacent connective tissues: $H R=3.37, P \otimes 0.05$; extension adjacent organs and structures: $H R=5.25$, $P<0.001$; tumor further contiguous extension: $H R=5.61, P<0.001)$, tumor size $(H R=0.95, P<0.001)$, Sex (female: $H R=0.59, P<0.1)$, and year of diagnosis ( $H R=0.96, P<0.01)$. Race (Others: $H R=1.14, P>0.1)$ and laterality (paired site: $H R=5.44, P>0.1$ ) were not classified as the independent prognostic factors. Moreover, the Kaplan-Meier curves were used to show the effect of prognostic factors on the survival of patients (Figure 4).

\subsection{Prognostic nomogram of overall survival}

The predictive model was developed and optimized according to the results of the AIC protocol in the primary cohort, and then visualized by the prognosis nomogram (Figure 5). Each level of every variable match corresponding scores assigned in points on the nomogram. Consequently, a total score can be obtained by adding the various variables or their corresponding levels. At this point, it is easy to estimate 
the overall survival of per patient at 3,5, and 10 years based on a total score. It is worth noting that the extent of tumor extension holds a greater weight of total score than other factors, indicating that extent of tumor extension is a chief factor influencing the prognosis of patients with PB.

\subsection{Validation of the nomogram}

The validation cohort with data of 71 PB patients was applied to validate the nomogram which exhibited an excellent consistency with a C index of 0.805 (95\% Cl: 0.78-0.83). In addition, the internal and external validation, as well as cross-validation of the nomogram was visualized by the calibration plots (figures 6A-F, figure 7A) in primary cohorts, validation cohorts, and both cohorts, respectively. Importantly, those plots exhibited an excellent consistency between observation and prediction for probability for 3-, 5-, and 10-year survival. Besides, the good discriminative ability of the model was demonstrated by the timedependent ROC curve (figures 7B) with the area under the curve (AUC) ranged from 0.7 to 0.9. Finally, the clinical usefulness was assessed by the DCA curves, in detail, a model curve between the blue and green line signifies that the model holds good clinical usefulness. Of note, if there is a more distance from the model curves to the blue and green line, which implies more significant net benefit and better clinical applicability. As is shown in figure 8, great net benefits were exhibited on the DCA curves of this predictive model (figures $8 \mathrm{~A}-\mathrm{C}$ ), which underlined the potential clinical utility of this nomogram.

\subsection{Comparison of predictive accuracy between the nomogram and a single independent factor}

Compared with other factors, the greater weights were found in the factors extension and year of diagnosis in the nomogram. The predictive ability for survival probability of patients with PB was, thus, compared between the nomogram and extent of tumor extension, as well as the year of diagnosis. Interestingly, there is a significantly higher C-index (0.805; P凶0.01) in nomogram than that in the extent of tumor extension (0.63) and year of diagnosis (0.59), indicating that there is greater accuracy in nomogram than the extent of tumor extension and year of diagnosis.

\section{Discussion}

It is of great importance for rare disorders such as PB to identify the prognostic factors affecting the survival of patients and evaluate the effectiveness of current treatment regimens. Thus, acquiring abundant clinical materials of PB cases is critical to achieving this goal. However, to conduct prospective clinical trials seem an exceedingly challenging issue owing to limited PB cases. In this study, we conducted a retrospective analysis based on enormous clinical data of PB which was collected by the SEER database from 1976 to 2017. To the best of our knowledge, this study was the largest series analysis concerning PB. Notably, the results from this analysis confirmed that age, year of diagnosis, treatment options, the extent of tumor extension, and tumor size were the dependent prognosis of patients with PB. Interestingly, the results of this analysis indicated that the overall survival rate of patients with PB has little to do with the application of radiotherapy. However, the combination of RT and CT was found to be an independent prognostic factor for survival. 
Hitherto, there is no defining the optimal management strategies for PB, owing to extremely limited data. Furthermore, clinical risk factors associated with prognosis were still less clear in groups with PB. Thus the aim of the study was to clear the ideal treatment regimen and identify the independent prognostic factors for PB. Given a higher precision of nomogram developed previously than the classical stage system for prognosis in some cancers $(29,30)$, a prognostic nomogram for PB, consequently, was developed based on the data of 243 PB cases. Finally, the clinical utility of the nomogram was assessed by the DCA.

The aggressiveness, the major characteristic of malignancy, is the chief cause of most cancer patients with a poor prognosis. The results of this study revealed, almost without exception, that the overriding risk factor of PB was the continuous extension of disease, which was in line with those studies $(31,32)$. Detailed, the HR for groups with tumor extension extended to adjacent connective tissues versus those with tumors confined to the gland of origin was 3.37, while that for tumor extension extended to adjacent organs or structures versus those with tumors confined to the gland of origin was 5.25 . The finding indicated that the death risk of groups with tumor extension extended to adjacent organs or structures was roughly four-fold of those with tumors confined to the gland of origin. As well, survival analysis demonstrated that overall survival was, compared with those whom tumors confined to the gland of origin, much reduced in groups whose tumor extension exceeded the gland of origin.

Thus far, the impact of tumor size on prognosis is still an intensely controversial issue. The results of a study conducted by Tirada and her colleagues found that increased tumor size often portended a poor prognosis for patients with cancers (33). Consistent with this, a study from Dittmar et al. showed that one of the high-risk factors for mortality was the larger tumor size (34). Besides, it has been reported that the risk of death and recurrence was increased as enlargement of tumor size (35). Yet there is evidence that tumor patients trended to a more poor survival when tumor size was smaller $(36,37)$. In conformity with this, the results of this analysis revealed that smaller tumor size was an independent prognostic factor of poor survival $(H R=0.95, P \unrhd 0.01)$, which indicated that increased risk of death by $5 \%$ for every $1 \mathrm{~mm}$ decrease in tumor size.

Multiple lines of evidence illustrated that PB, comparing to infratentorial primitive neuro-ectodermal tumors, carried more poor outcomes irrespective of treatment regimens (38-40). But the aggressive surgical resection is still recommended in the management of PB, and surgical treatment was regarded as the first-line therapy strategy $(9,20)$. In this analysis, the effect of varied treatment regimens on the prognosis of patients with PB was analyzed, and found that those treatment options presented different influences on clinical outcomes. It is important to note that the risk of death was diminished considerably in patients who received the RT combined with CT, RT after surgical treatment as well as RT after surgery combined with CT. Precisely, the HR for the RT combined with CT versus RT was 0.17 , while that for RT after surgical treatment and RT after surgery combined with CT versus CT was 0.38 and 0.30 , respectively. Those results indicated that the risk of death is reduced by $62 \%$ and $70 \%$ in patients who underwent RT after surgery and in patients who underwent RT after surgery combined CT, respectively, compared with those who underwent CT alone. Unsurprisingly, survival analysis in this study draws a 
conclusion that was in line with those findings. Though surgical resection was known as the first-line therapeutic option and strongly recommended (21), of note, the results of this study could not yet support that surgical treatment alone could significantly improve the prognosis of patients with PB. Interestingly, the results from a survey conducted by Mallick and his colleagues revealed that the clinical outcome was markedly improved in patients who received surgical resection and adjuvant radiotherapy (41). The findings of this study found that the RT after surgical resection and RT after surgery combined with CT were independent prognostic factors which could improve remarkably clinical survival outcome.

About the function of CT in PB is a contentious issue. It seems that PB was responsive to CT as reported by Schild et al. (42) and Ghim et al. (43). However, the findings of this study found that CT failed to improve the overall survival of patients, which was not in harmony with that. It is interesting to note that a study conducted by Mynarek et al. (6) draws a similar conclusion with us, which could not support the effectiveness of CT in PB. Likewise, there is also no consensus regarding the role of RT against PB. A study by Clark and his colleagues (44) demonstrated that PB was resistant to the RT. However, several studies believed that PB was sensitive to the ratio therapy $(39,45,46)$. But the findings of this analysis could not determine that patients only received RT was benefitting from the RT treatment. The limited number of PB cases may be the primary reason why the discrepancy occurred on the effectiveness of RT and CT. Hence, high-quality clinical studies are crucial to clarify those disagreements about RT and CT.

Age is typically regarded as risk factors of many disorders, such as neurodegenerative disease.

It was of interest that age was also a key risk factor of PB, which was identified in this analysis. Our findings revealed that overall survival improved significantly with the increase of age $(H R=0.73)$, which was in agreement with studies (47-49). In great detail, the risk of death was reduced by $27 \%$ for every 5 year increase in patient age. It may perhaps be the fact that the biological behaviors of PB vary with increasing age, but it is essential that plenty of studies were conducted to confirm this conclusion.

There was no doubt that this study also has some limitations. Firstly, a long span of a few decades' years consumed patient data collection leads to heterogeneity in an examination, diagnosis, and treatment modalities. Additionally, part of the information on some critical items of tumor size and extension was not available in the primary data in which multiple imputation was performed. Although multiple imputation adopted widely in some studies was conducted in this analysis, there is inevitably an existing difference between the primary data and imputation data. Thirdly, the sequence of various treatment options was unknown, which might lead to an unrealized evaluation for the effect of the sequence on patients' prognosis. Despite the shortcomings of this study, its advantages were also considerable and conspicuous. Compared with previous studies, more abundant information about prognostic factors of PB was supplied in this study. Also, the inherent bias and heterogeneity were limited through the regression analysis, and the statistical power was improved significantly on account of enlargement in the number of PB cases. More importantly, the risk factors in the model were more accessible to achieve than those realized by a high-priced technique and a great deal of time. 


\section{Conclusions}

In summary, age, year of diagnosis, treatment approaches, tumor extension, and tumor size were identified as independent prognostic factors of PB in this study. Additionally, we developed and validated a nomogram that presented an accurate and nonsubjective prediction for the prognosis of patients with PB. Most importantly, the nomogram may act as an assistant that helps clinicians assess patients' prognosis and therapy decisions.

\section{Abbreviations}

PB: pineoblastoma

C-index: concordance index

ROC: receiver operating characteristic

DCA: decision curve analysis

AUC: area under the curve

PPT: pineal parenchymal tumor

SEER database: Surveillance, Epidemiology,and End Results database

ICD-0-3: International Classification of Diseasesfor Oncology, third Edition; WHO, World Health Organization

BRT: beam radiotherapy

CT: chemotherapy

GTR: gross total resection

\section{Declarations}

\section{Availability of data and materials}

This data can be found in SEER Database (https://seer.cancer.gov/data/).

\section{Acknowledgments}

Not Applicable

\section{Funding}


This work was supported by the Key Research and Development Project of Shandong (Grant Number 2015GSF118177).

\section{Ethics declarations}

The SEER Research Data Agreement to protect the privacy of the patients was signed by the authors, which is consistent with ethical principles.

Competing Interest

All authors report no any conflicts of interestin this study.

Consent for publication

Not applicable.

\section{References}

1. JY. Lee, T. Wakabayashi, J. Yoshida, Management and survival of pineoblastoma: an analysis of 34 adults from the brain tumor registry of Japan, Neurologia medico-chirurgica, 45 (2005) 132-141; discussion 141-132.

2. H.J. Hoffman, M. Yoshida, L.E. Becker, E.B. Hendrick, R.P. Humphreys, Pineal region tumors in childhood. Experience at the Hospital for Sick Children. 1983, Pediatric neurosurgery, 21 (1994) 91103; discussion 104.

3. E.R. Wilson, Y. Takei, W.T. Bikoff, M.S. O'Brien, G.T. Tindall, W.M. Boehm, Abdominal metastases of primary intracranial yolk sac tumors through ventriculoperitoneal shunts: report of three cases, Neurosurgery, 5 (1979) 356-364.

4. D.N. Louis, A. Perry, G. Reifenberger, A. von Deimling, D. Figarella-Branger, W.K. Cavenee, H. Ohgaki, O.D. Wiestler, P. Kleihues, D.W. Ellison, The 2016 World Health Organization Classification of Tumors of the Central Nervous System: a summary, Acta neuropathologica, 131 (2016) 803-820.

5. B. Farnia, P.K. Allen, P.D. Brown, S. Khatua, N.B. Levine, J. Li, M. Penas-Prado, A. Mahajan, A.J. Ghia, Clinical outcomes and patterns of failure in pineoblastoma: a 30-year, single-institution retrospective review, World neurosurgery, 82 (2014) 1232-1241.

6. M. Mynarek, B. Pizer, C. Dufour, D. van Vuurden, M. Garami, M. Massimino, J. Fangusaro, T. Davidson, M.J. Gil-da-Costa, J. Sterba, M. Benesch, N. Gerber, B.O. Juhnke, R. Kwiecien, T. Pietsch, M. Kool, S. Clifford, D.W. Ellison, F. Giangaspero, P. Wesseling, F. Gilles, N. Gottardo, J.L. Finlay, S. Rutkowski, K. von Hoff, Evaluation of age-dependent treatment strategies for children and young adults with pineoblastoma: analysis of pooled European Society for Paediatric Oncology (SIOP-E) and US Head Start data, Neuro-oncology, 19 (2017) 576-585.

7. M.C. Tate, M.J. Rutkowski, A.T. Parsa, Contemporary management of pineoblastoma, Neurosurgery clinics of North America, 22 (2011) 409-412, ix. 
8. H. Mena, E.J. Rushing, J.L. Ribas, B. Delahunt, W.F. McCarthy, Tumors of pineal parenchymal cells: a correlation of histological features, including nucleolar organizer regions, with survival in 35 cases, Human pathology, 26 (1995) 20-30.

9. Y. Tian, R. Liu, J. Qin, J. Wang, Z. Ma, J. Gong, C. Li, Retrospective analysis of the clinical characteristics, therapeutic aspects, and prognostic factors of 18 cases of childhood pineoblastoma, World neurosurgery, 116 (2018) e162-e168.

10. F. Gaillard, J. Jones, Masses of the pineal region: clinical presentation and radiographic features, Postgraduate medical journal, 86 (2010) 597-607.

11. S. Villa, R.C. Miller, M. Krengli, H. Abusaris, B.G. Baumert, S. Servagi-Vernat, S. Igdem, A. Lucas, S. Boluda, RO. Mirimanoff, Primary pineal tumors: outcome and prognostic factors-a study from the Rare Cancer Network (RCN), Clinical \& translational oncology : official publication of the Federation of Spanish Oncology Societies and of the National Cancer Institute of Mexico, 14 (2012) 827-834.

12. A.K. Banerjee, V.K. Kak, Pineoblastoma with spontaneous intra and extracranial metastasis, The Journal of pathology, 114 (1974) 9-12.

13. D. Golbin, KV Nikitin, A.N. Konovalov, D.I. Pitskhelauri, L.V. Shishkina, A.V. Golanov, V.A. Cherekaev, G.L. Kobiakov, O.V. Absalyamova, N. Lasunin, N. Antipina, Intraosseous metastasizing of pineoblastoma into the anterior skull base, calvarial bones, and vertebrae, Cureus, 7 (2015) e437.

14. E. Charafe-Jauffret, G. Lehmann, F. Fauchon, J.F. Michiels, P. Paquis, D. Maraninchi, J. Hassoun, Vertebral metastases from pineoblastoma, Archives of pathology \& laboratory medicine, 125 (2001) 939-943.

15. P. Zhao, A. Strohl, C. Gonzalez, T. Fishbein, S. Rosen-Bronson, B. Kallakury, M. Ozdemirli, Donor transmission of pineoblastoma in a two-yr-old male recipient of a multivisceral transplant: a case report, Pediatric transplantation, 16 (2012) E110-114.

16. G. Fraser, R. Rampling, C. Smith, J. Nicoll, M. Stephen, Long-term survival following extra-neural metastasis from a pineoblastoma, Journal of neuro-oncology, 48 (2000) 141-144.

17. P.E. Cranston, M.T. Hatten, E.E. Smith, Metastatic pineoblastoma via a ventriculoperitoneal shunt: CT demonstration, Computerized medical imaging and graphics : the official journal of the Computerized Medical Imaging Society, 16 (1992) 349-351.

18. N. Ghosal, S.V. Furtado, A.S. Hegde, Pinealoblastoma with prominent retinoblastic differentiation: an unusual case in an adult, Neuropathology : official journal of the Japanese Society of Neuropathology, 30 (2010) 439-442.

19. T. Gadish, H. Tulchinsky, A.A. Deutsch, M. Rabau, Pinealoblastoma in a patient with familial adenomatous polyposis: variant of Turcot syndrome type 2? Report of a case and review of the literature, Diseases of the colon and rectum, 48 (2005) 2343-2346.

20. M. Tate, M.E. Sughrue, M.J. Rutkowski, A.J. Kane, D. Aranda, L. McClinton, L. McClinton, I.J. Barani, A.T. Parsa, The long-term postsurgical prognosis of patients with pineoblastoma, cancer, 118 (2012) 173-179. 
21. M. Resche-Rigon, I.R. White, Multiple imputation by chained equations for systematically and sporadically missing multilevel data, Statistical methods in medical research, 27 (2018) 1634-1649.

22. Jing Y, Deng W, Zhang H, Jiang Y, Dong Z, Fan F and Sun P, Development and Validation of a Prognostic Nomogram to Predict Cancer-Specific Survival in Adult Patients With Pineoblastoma. Front. Oncol,10(2020)1021.

23. F.E. Harrell, Jr., K.L. Lee, D.B. Mark, Multivariable prognostic models: issues in developing models, evaluating assumptions and adequacy, and measuring and reducing errors, Statistics in medicine, 15 (1996) 361-387.

24. J.A. Hanley, B.J. McNeil, The meaning and use of the area under a receiver operating characteristic (ROC) curve, Radiology, 143 (1982) 29-36.

25. M. Wolbers, M.T. Koller, J.C. Witteman, E.W. Steyerberg, Prognostic models with competing risks: methods and application to coronary risk prediction, Epidemiology (Cambridge, Mass.), 20 (2009) 555-561.

26. C. Coutant, C. Olivier, E. Lambaudie, E. Fondrinier, F. Marchal, F. Guillemin, N. Seince, V. Thomas, J. Leveque, E. Barranger, E. Darai, S. Uzan, G. Houvenaeghel, R. Rouzier, Comparison of models to predict nonsentinel lymph node status in breast cancer patients with metastatic sentinel lymph nodes: a prospective multicenter study, Journal of clinical oncology : official journal of the American Society of Clinical Oncology, 27 (2009) 2800-2808.

27. A.J. Vickers, E.B. Elkin, Decision curve analysis: a novel method for evaluating prediction models, Medical decision making : an international journal of the Society for Medical Decision Making, 26 (2006) 565-574.

28. RL Camp, M. Dolled-Filhart, D.L. Rimm, X-tile: a new bio-informatics tool for biomarker assessment and outcome-based cut-point optimization, Clinical cancer research : an official journal of the American Association for Cancer Research, 10 (2004) 7252-7259.

29. S. Chen, Y. Liu, J. Yang, Q. Liu, H. You, Y. Dong, J. Lyu, Development and validation of a nomogram for predicting survival in male patients with breast cancer, Frontiers in oncology, 9 (2019) 361.

30. A. Martini, S. Cumarasamy, A.T. Beksac, R. Abaza, DD Eun, A. Bhandari, A.K. Hemal, J.R. Porter, K.K. Badani, A nomogram to predict significant estimated glomerular filtration rate reduction after robotic partial nephrectomy, European urology, 74 (2018) 833-839.

31. J. Lutterbach, F. Fauchon, S.E. Schild, S.M. Chang, A. Pagenstecher, B. Volk, C. Ostertag, F. Momm, A. Jouvet, Malignant pineal parenchymal tumors in adult patients: patterns of care and prognostic factors, Neurosurgery, 51 (2002) 44-55; discussion 55-46.

32. RI. Jakacki, P.M. Zeltzer, J.M. Boyett, A.L. Albright, J.C. Allen, J.R. Geyer, L.B. Rorke, P. Stanley, K.R. Stevens, J. Wisoff, et al., Survival and prognostic factors following radiation and/or chemotherapy for primitive neuroectodermal tumors of the pineal region in infants and children: a report of the Childrens Cancer Group, Journal of clinical oncology : official journal of the American Society of Clinical Oncology, 13 (1995) 1377-1383. 
33. Y. Dittmar, M. Ardelt, H. Scheuerlein, F. Rauchfuss, F. Dondorf, U. Settmacher, The Impact of Tumor Size on the Prognosis of Gastric Cancer: Experiences from a European Study Group, J Surg., 3 (2016) 1082.

34. JY. Park, D.Y. Kim, J.H. Kim, Y.M. Kim, Y.T. Kim, J.H. Nam, Outcomes after radical hysterectomy according to tumor size divided by 2-cm interval in patients with early cervical cancer, Annals of oncology: official journal of the European Society for Medical Oncology, 22 (2011) 59-67.

35. B. Huang, Y. Feng, S.B. Mo, S.J. Cai, L.Y. Huang, Smaller tumor size is associated with poor survival in T4b colon cancer, World journal of gastroenterology, 22 (2016) 6726-6735.

36. H.-S. Han, H.-J. Paik, J.M. Ryu, S. Park, H.W. Yi, S. Bae, S. Lee, W.H. Kil, J.E. Lee, S.J. Nam, S.W. Kim, Comparison of prognosis and specific features according to tumor size in small-sized breast cancer with extensive lymph node involvement, Journal of Clinical Oncology, 33 (2015) 81-81.

37. N. Tirada, M. Aujero, G. Khorjekar, S. Richards, J. Chopra, S. Dromi, O. loffe, Breast Cancer Tissue Markers, Genomic Profiling, and Other Prognostic Factors: A Primer for Radiologists, Radiographics: a review publication of the Radiological Society of North America, Inc, 38 (2018) 1902-1920.

38. A.T. Reddy, A.J. Janss, PC. Phillips, H.L. Weiss, R.J. Packer, Outcome for children with supratentorial primitive neuroectodermal tumors treated with surgery, radiation, and chemotherapy, cancer, 88 (2000) 2189-2193.

39. S. Gururangan, C. McLaughlin, J. Quinn, J. Rich, D. Reardon, E.C. Halperin, J. Herndon, 2nd, H. Fuchs, T. George, J. Provenzale, M. Watral, R.E. McLendon, A. Friedman, H.S. Friedman, J. Kurtzberg, J. Vredenbergh, P.L. Martin, High-dose chemotherapy with autologous stem-cell rescue in children and adults with newly diagnosed pineoblastomas, Journal of clinical oncology : official journal of the American Society of Clinical Oncology, 21 (2003) 2187-2191.

40. SW. Gilheeney, A. Saad, S. Chi, C. Turner, N.J. Ullrich, L. Goumnerova, R.M. Scott, K. Marcus, L. Lehman, U. De Girolami, M.W. Kieran, outcome of pediatric pineoblastoma after surgery, radiation and chemotherapy, Journal of neuro-oncology, 89 (2008) 89-95.

41. S. Mallick, R. Benson, G.K. Rath, Patterns of care and survival outcomes in patients with pineal parenchymal tumor of intermediate differentiation: An individual patient data analysis, radiotherapy and oncology: journal of the European Society for Therapeutic Radiology and Oncology, 121 (2016) 204-208.

42. S.E. Schild, B.W. Scheithauer, P.J. Schomberg, C.C. Hook, P.J. Kelly, L. Frick, J.S. Robinow, S.J. Buskirk, Pineal parenchymal tumors. Clinical, pathologic, and therapeutic aspects, cancer, 72 (1993) 870-880.

43. T.T. Ghim, P. Davis, J.J. Seo, I. Crocker, M. O'Brien, N. Krawiecki, Response to neoadjuvant chemotherapy in children with pineoblastoma, cancer, 72 (1993) 1795-1800.

44. A.J. Clark, M.E. Sughrue, M.E. Ivan, D. Aranda, M.J. Rutkowski, A.J. Kane, S. Chang, A.T. Parsa, Factors influencing overall survival rates for patients with pineocytoma, Journal of neuro-oncology, 100 (2010) 255-260.

45. M. Massimino, L. Gandola, F. Spreafico, R. Luksch, P. Collini, F. Giangaspero, F. Simonetti, M. Casanova, G. Cefalo, E. Pignoli, A. Ferrari, M. Terenziani, M. Podda, C. Meazza, D. Polastri, G. Poggi, F. 
Ravagnani, F. Fossati-Bellani, Supratentorial primitive neuroectodermal tumors (S-PNET) in children: A prospective experience with adjuvant intensive chemotherapy and hyperfractionated accelerated radiotherapy, International journal of radiation oncology, biology, physics, 64 (2006) 1031-1037.

46. B. Timmermann, R.D. Kortmann, J. Kuhl, C. Meisner, K. Dieckmann, T. Pietsch, M. Bamberg, Role of radiotherapy in the treatment of supratentorial primitive neuroectodermal tumors in childhood: results of the prospective German brain tumor trials HIT 88/89 and 91,Journal of clinical oncology: official journal of the American Society of Clinical Oncology,20 (2002) 842-849.

47. K.A. Parikh, G.T. Venable, B.A. Orr, A.F. Choudhri, F.A. Boop, A.J. Gajjar, P. Klimo, Jr., PineoblastomaThe Experience at St. Jude Children's Research Hospital, Neurosurgery, 81(2017) 120-128.

48. M. Tate, M.E. Sughrue, M.J. Rutkowski, A.J. Kane, D. Aranda, L. McClinton, L. McClinton, I.J. Barani, A.T. Parsa, The long-term postsurgical prognosis of patients with 30 pineoblastoma, cancer, 118 (2012) 173-179.

49. M.C. Jin, L.M. Prolo, A. Wu, T.D. Azad, S. Shi, A.J. Rodrigues, S.G. Soltys, E.L. Pollom, G. Li, S.M. Hiniker, G.A. Grant, Patterns of Care and Age-Specific Impact of Extent of Resection and Adjuvant Radiotherapy in Pediatric Pineoblastoma, Neurosurgery, 86 (2020)E426-e435.

\section{Tables}

Table 1. Characteristics and treatments information of all patients 


\section{Validation cohort primary} cohort

\section{Age}

$\otimes 5$

$21(29.58 \%) \quad 45(26.32 \%)$

5-14

$23(32.39 \%) \quad 56(32.75 \%)$

$15-29$

27 (38.02\%) 71 (41.52\%)

Sex

Male

$35(49.30 \%) \quad 83(48.26 \%)$

Female

$36(50.70 \%) \quad 89(51.74 \%)$

Race

White

$43(60.56 \%) \quad 120(69.77 \%)$

Others

$28(39.44 \%) \quad 52(30.23 \%)$

Year of diagnosis

1975-2002

$21(29.58 \%) \quad 65(37.79 \%)$

2003-2016

$50(70.42 \%)$

$107(62.21 \%)$

Tumor size

$29.74 \pm 10.03$ $30.79 \pm 10.94$

Laterality

Not a paired site

$70(98.59 \%) \quad 169(98.26 \%)$

Paired site

$1(1.41 \%)$

$3(1.74 \%)$

Therapy

Surgery

$4(5.63 \%) \quad 10(5.81 \%)$

RT

$3(4.23 \%) \quad 12(6.98 \%)$

Biopsy

$1(1.41)$

$5(2.91 \%)$

CT

$10(14.08 \%) \quad 15(8.72 \%)$

RT \& CT, sequence unknown

$4(5.63 \%) \quad 22(12.79 \%)$

RT after surgery

$9(12.68 \%) \quad 30(17.44 \%)$

RT after surgery \& CT, sequence unknown

$37(15.23 \%) \quad 67(27.57 \%)$

RT after GTR \& CT

$30(12.35 \%) \quad 53$

(21.81\%)

RT after STR \& CT

$7(2.88 \%) \quad 14(5.98 \%)$ 
RT after non-primary surgical procedure \& $C T$, sequence

\section{Extension}

Confined to the gland of origin

Localized, NOS

$$
\begin{array}{ll}
40(56.33 \%) & 92(53.49 \%) \\
15(21.13 \%) & 36(20.93 \%) \\
3(4.23 \%) & 7(4.07 \%) \\
13(18.31) & 28(16.28 \%) \\
1(1.41 \%) & 8(4.65 \%) \\
69(97.18 \%) & 170(98.83 \%) \\
1(1.41 \%) & 2(1.17 \%) \\
1(1.41 \%) & 0(0 \%)
\end{array}
$$

Adjacent connective tissues

Adjacent organs \& structures

Further continuous extension

\section{Diagnostic Confirmation}

Positive histology

Radiography

Positive exfoliative cytology

Note: paired site including right-origin of primary, lift-origin of primary, and paired site, but no information concerning laterality. RT, Beam radiotherapy; CT, chemotherapy; GTR, gross total resection; STR, Subtotal resection.

\section{Figures}




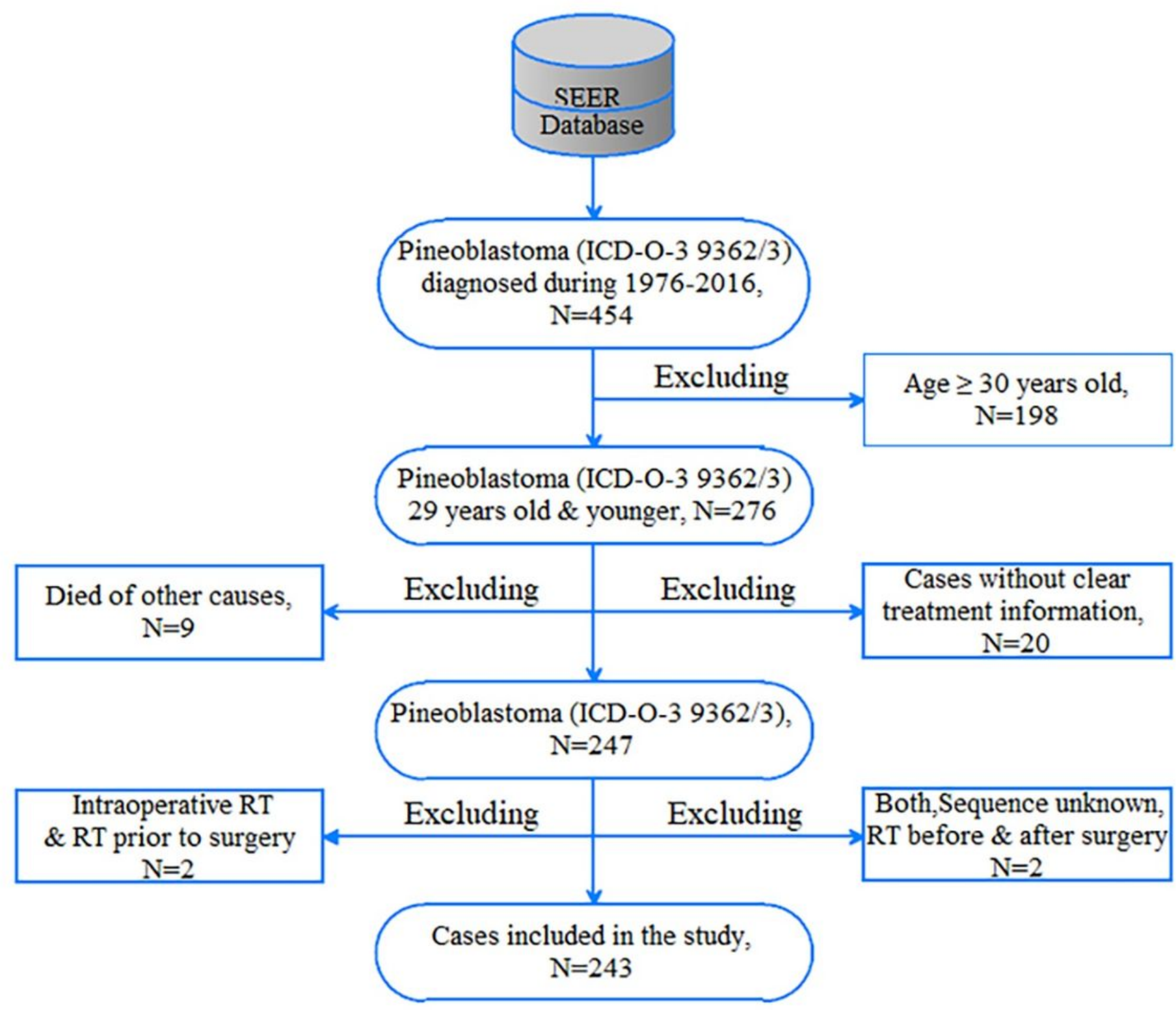

Figure 1

The flow chart of patient enrollment. 


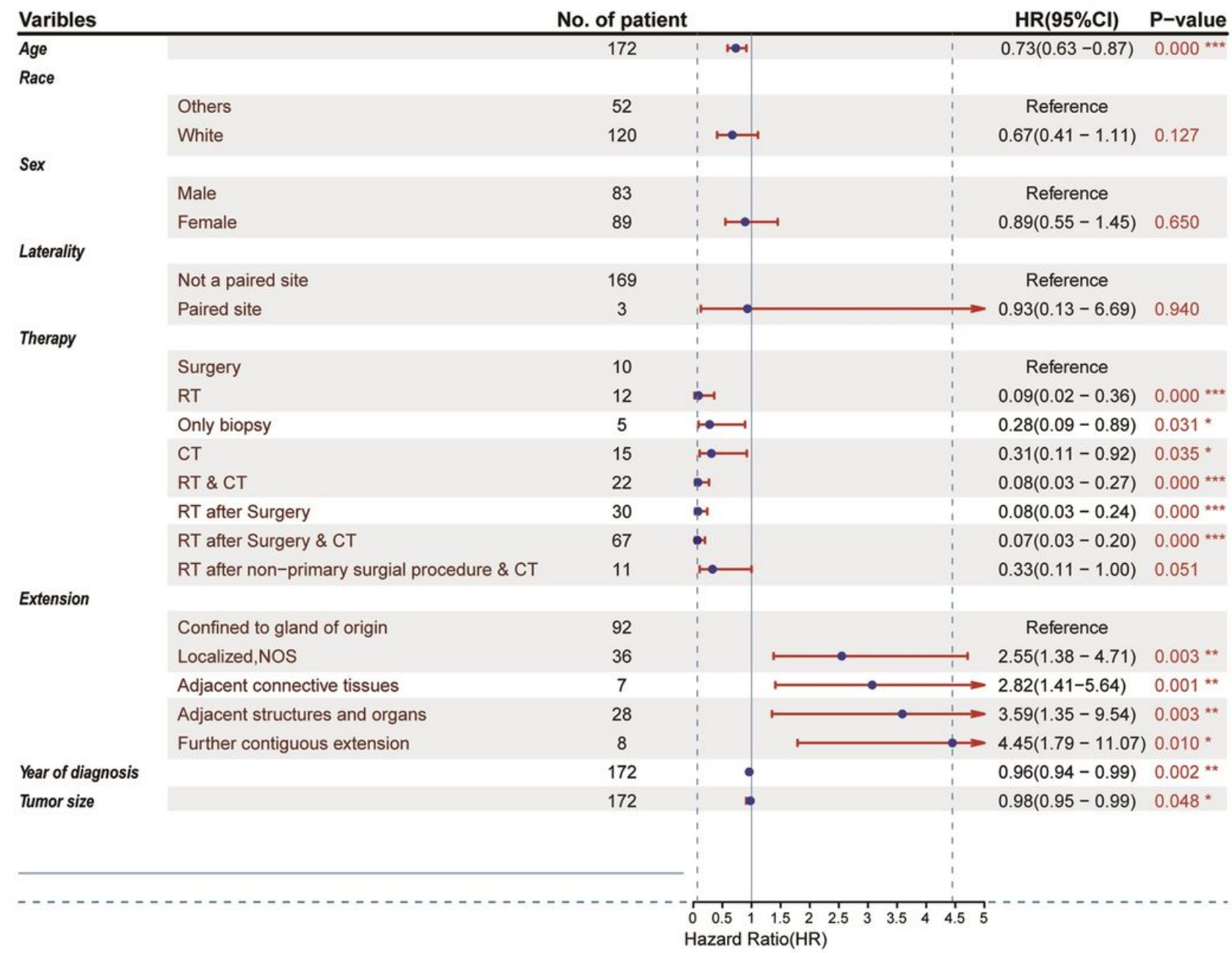

\section{Figure 2}

The results of the univariate Cox proportional hazards analysis (HR, 95\% confidence interval). Note: *means $\mathrm{p} \otimes 0.05,{ }^{*}$ means $\mathrm{p} \otimes 0.01$, and ${ }^{* * *}$ means $\mathrm{p} \otimes 0.001$. 


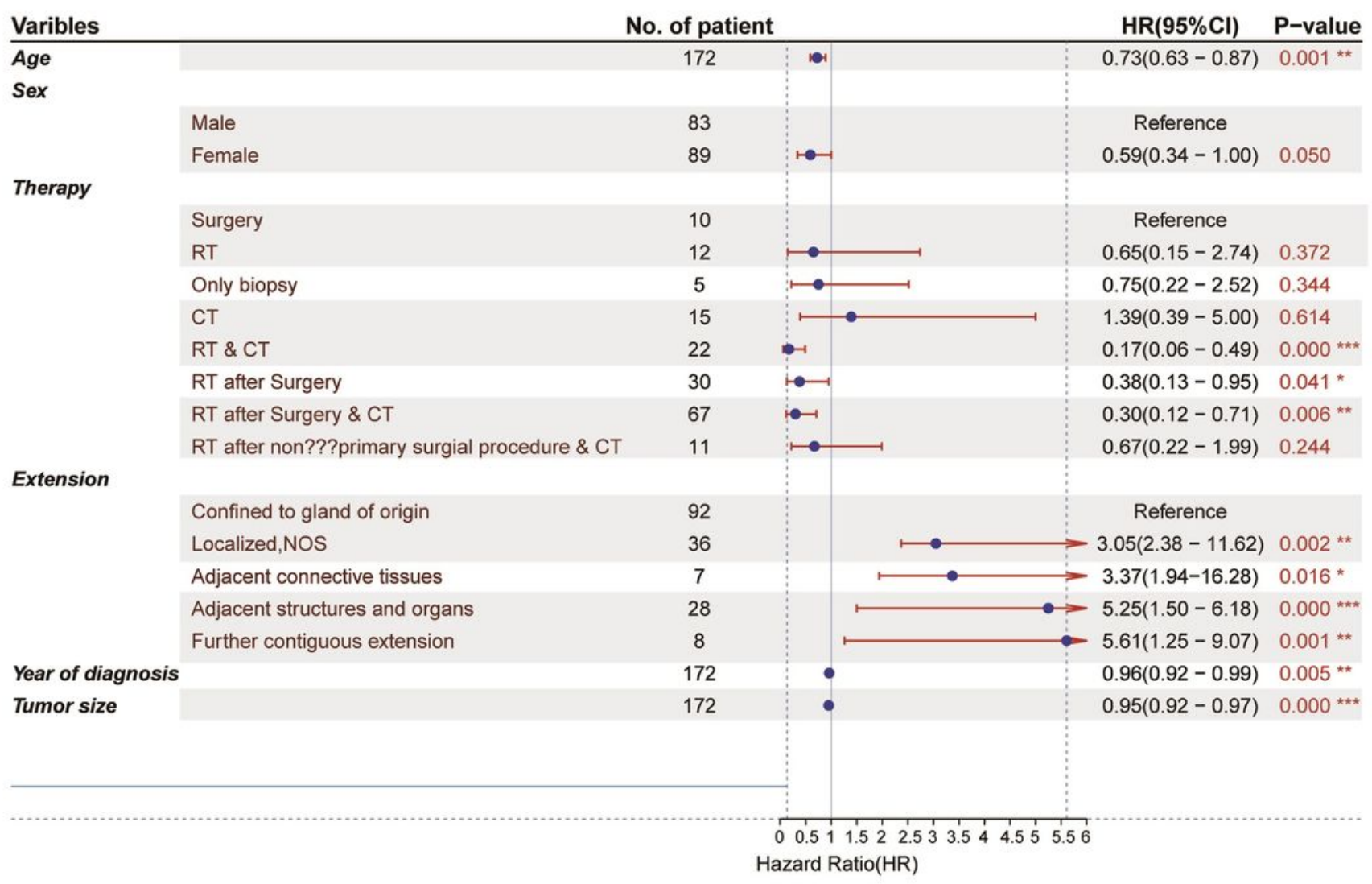

\section{Figure 3}

Results of the multivariate analysis of different factors (HR, 95\% confidence interval). Note: *means

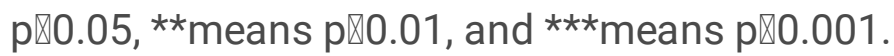



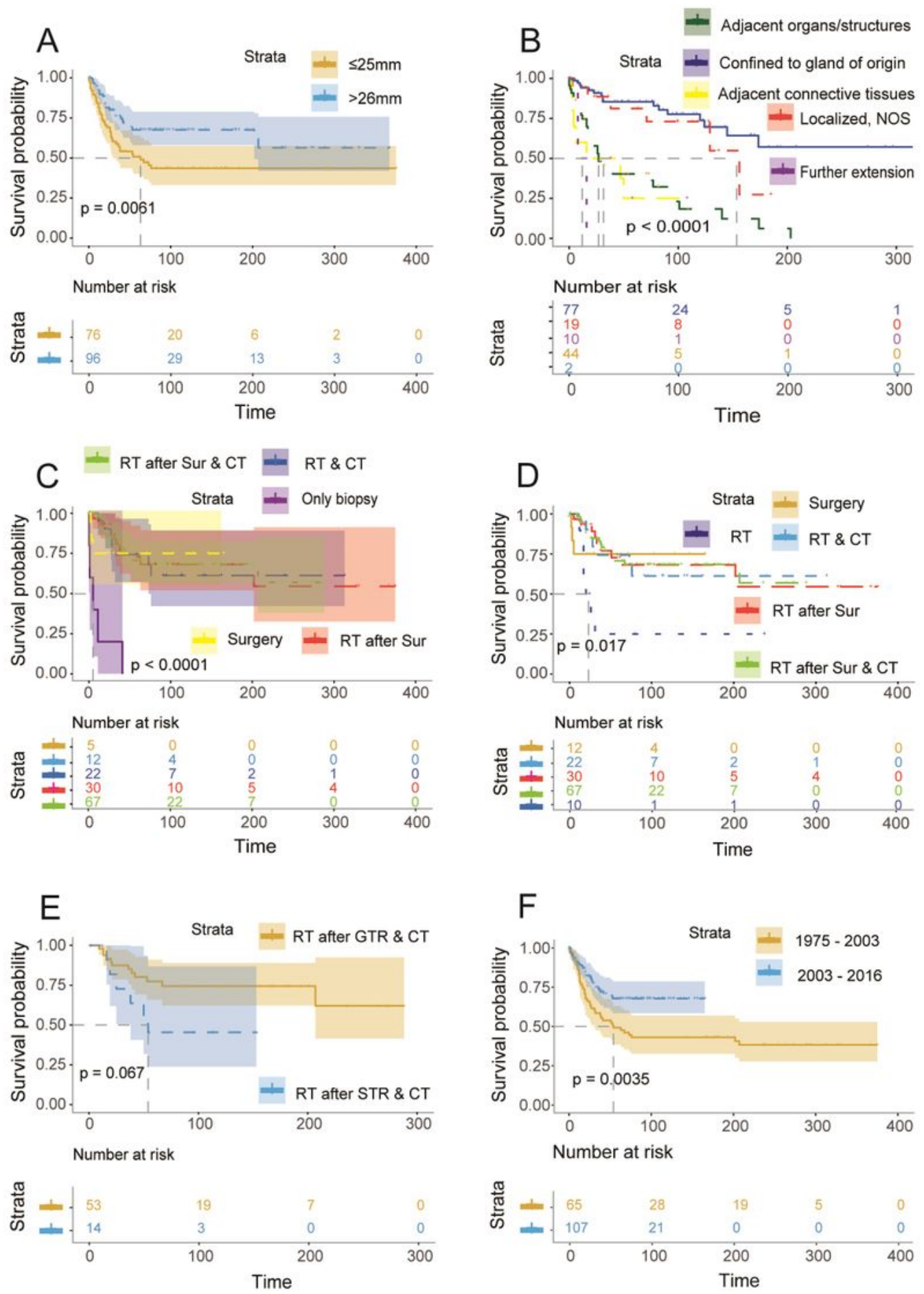

\section{Figure 4}

Kaplan-Meier curves for patients with PB based on varied independent prognostic factors. The KaplanMeier curves for patients with primary PB according to tumor size (A), the extent of extension (B), therapy \& biopsy (C), therapy (D), RT after GTR \& CT, RT after STR \& CT (E), years of diagnosis (F). 
Nomogram

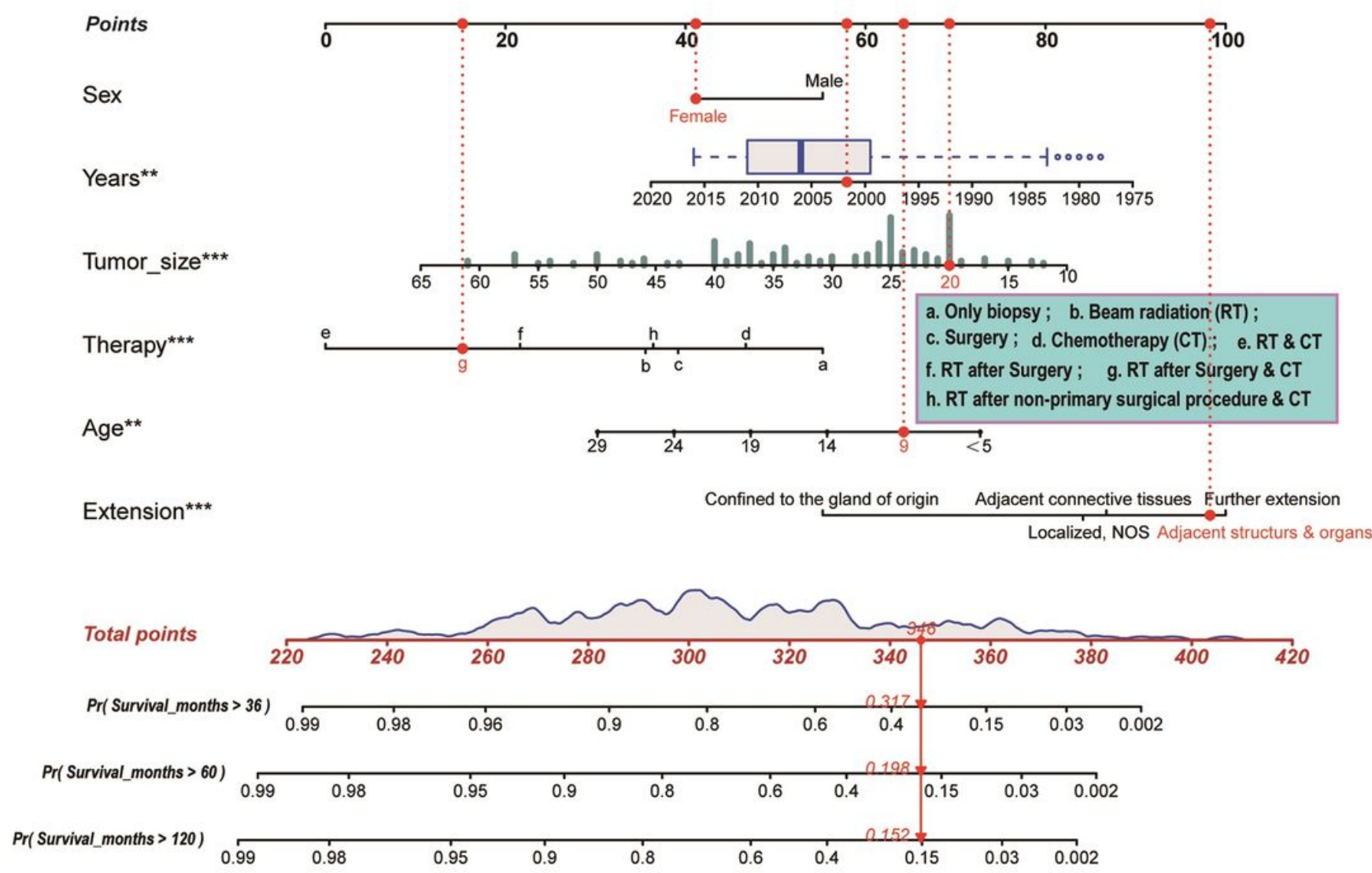

\section{Figure 5}

Nomogram predicting 3-, 5-, and 10-year cancer-specific survival for patients with PB. Prognostic factors including age, race, tumor extension, tumor size, and therapy, and the scores assigned on the points scale could match each level of every variable on the nomogram. Thus, a total score was obtained by adding the score from various variables or their levels. Finally, the 3-, 5-, and 10-year cancer-specific survival for each patient could be estimated based on the total score. 

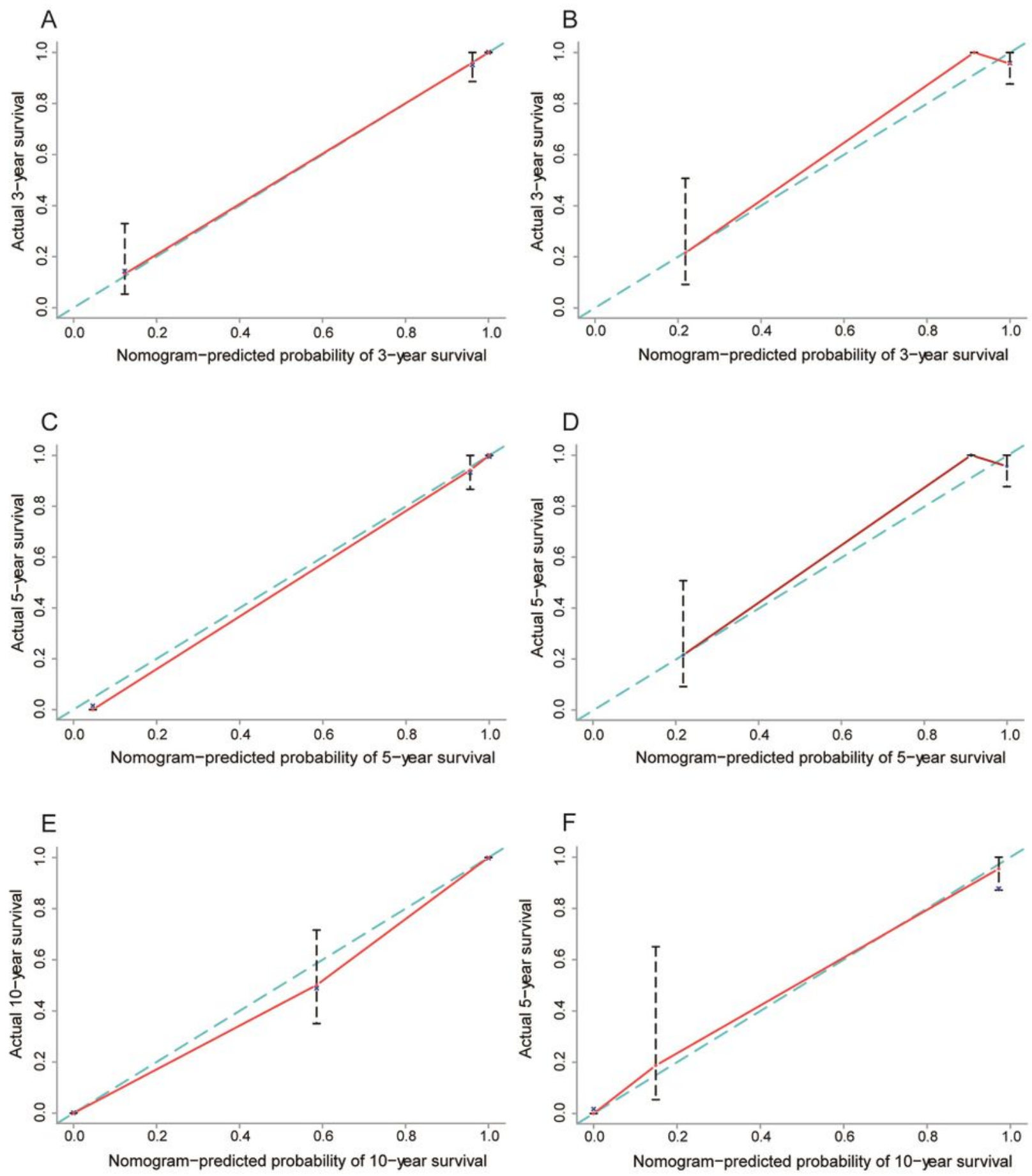

\section{Figure 6}

Calibration plots for the prediction of cancer-specific survival at 3-, 5-, and 10-months. (A-C) Calibration curves displaying the probability of 3-, 5-, and 10-year cancer-specific survival between the actual observation and the probability predicted by the nomogram in the primary cohort. (D-F) Calibration curves showing the probability of 3-,5-, and 10-year cancer-specific survival between the actual 
observation and the probability predicted by the nomogram in the validation cohort. The blue lines with a slope of 1 were ideal for prediction.
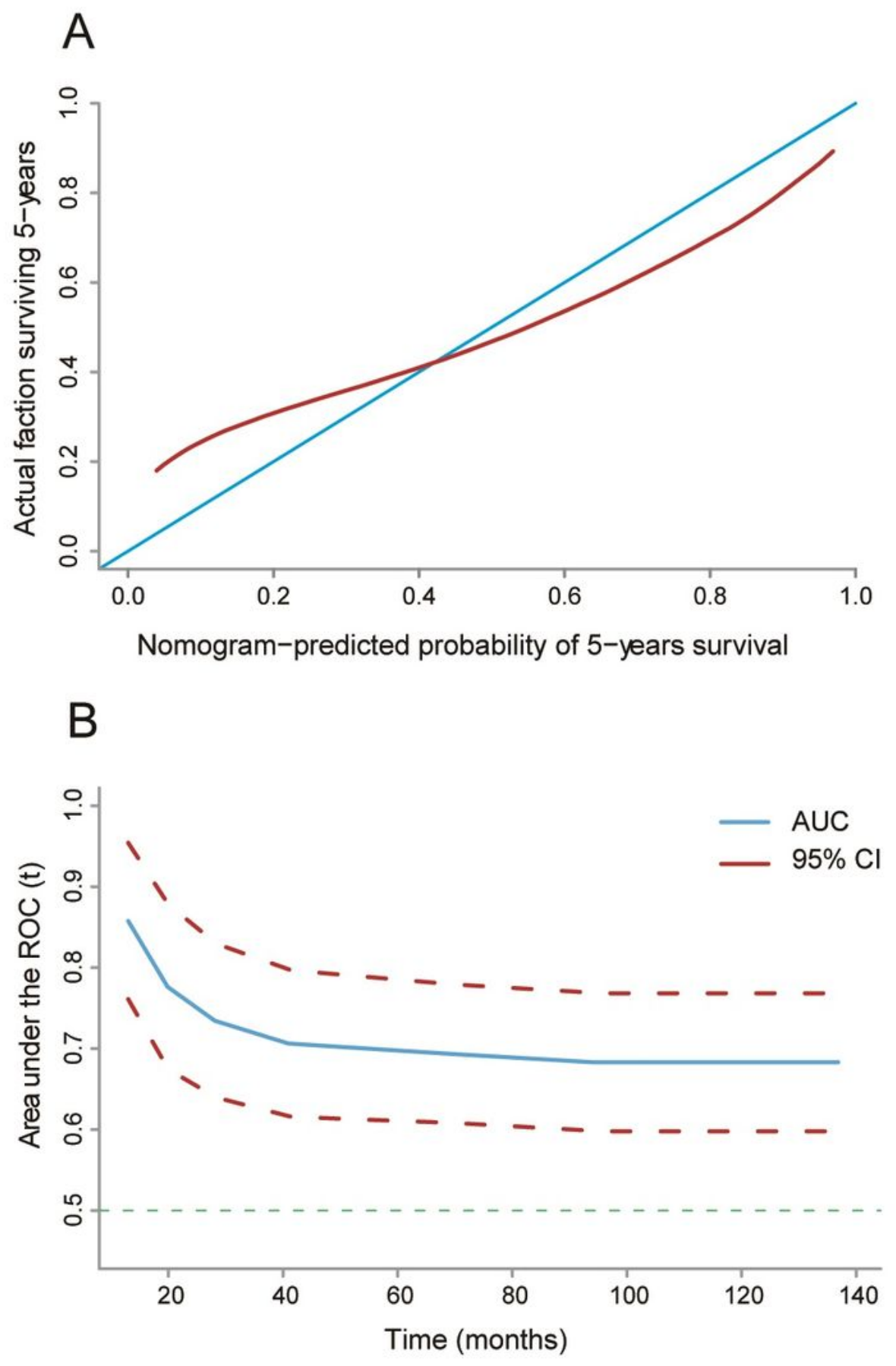

Figure 7

(A) Cross-validation curves showing the probability of 5-year specific survival between the actual observation and the probability predicted by the nomogram in the validation cohort. The blue lines with a 
slope of 1 were ideal for prediction. (B) time-dependent ROC curves showing the sensitivity and specificity of the cancer-specific survival prediction by the nomogram.
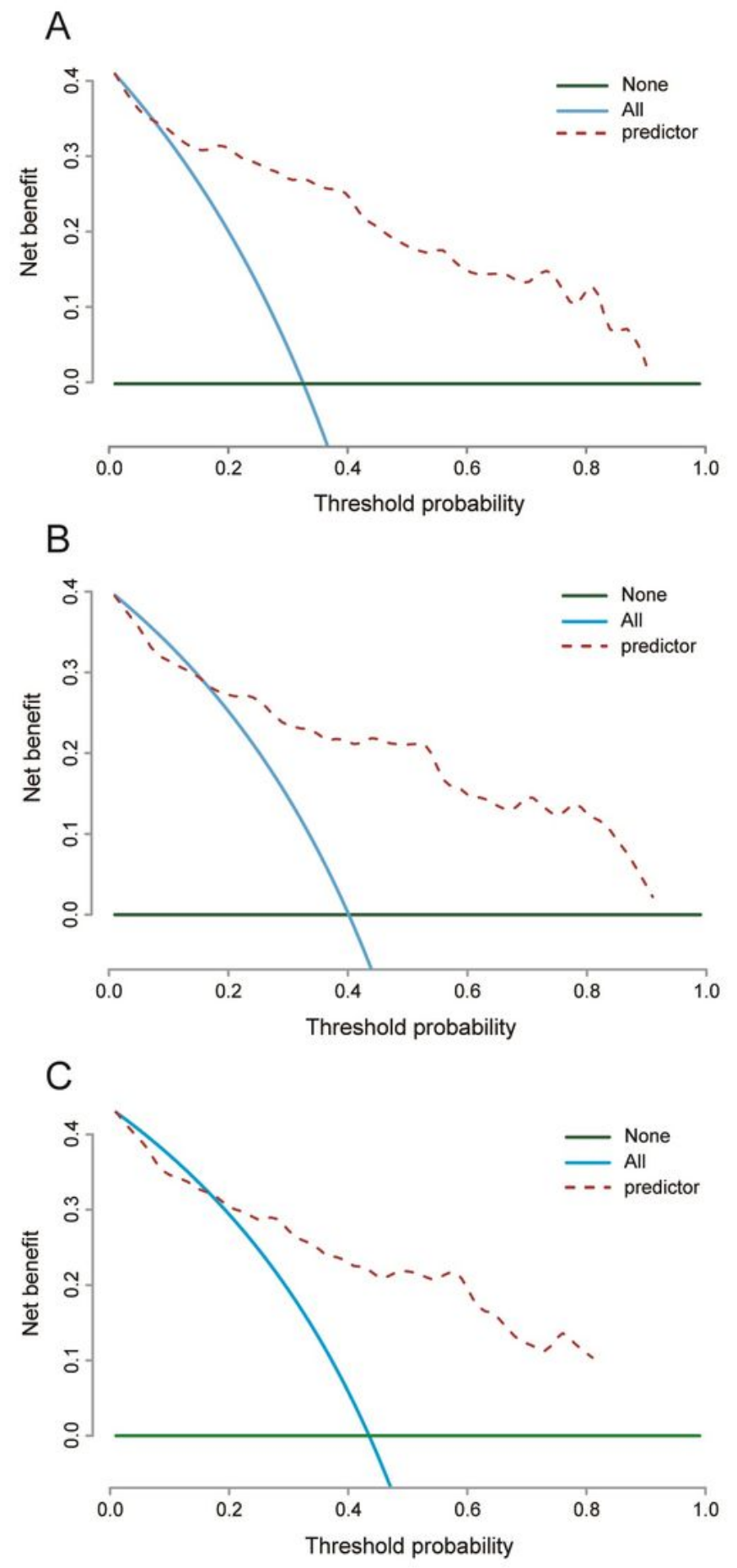

\section{Figure 8}

Decision curves of the nomogram predicting 3- (A), 5- (B), and 10-year(C) cancer-specific survival. The $x-$ axis represents the threshold probability, while the $y$-axis indicates the net benefit calculated by adding the true positives and subtracting the false positives. The horizontal line in parallel with the $x$-axis 
assumes that cancer-specific death did not occur in any of the patients, while the solid green line assumes that all patients will have cancer-specific death at a specific threshold probability. The brown dashed line represents the net benefit of using the nomogram. 